\title{
Highly Variable Spike Trains Underlie Reproducible Sensorimotor Responses in the Medicinal Leech
}

\author{
Davide Zoccolan, Giulietta Pinato, and Vincent Torre \\ Scuola Internazionale Superiore di Studi Avanzati and Istituto Nazionale di Fisica della Materia, 34014 Trieste, Italy
}

\begin{abstract}
The nervous system of the leech is a particularly suitable model to investigate neural coding of sensorimotor responses because it allows both observation of behavior and the simultaneous measurement of a large fraction of its underlying neuronal activity. In this study, we used a combination of multielectrode recordings, videomicroscopy, and computation of the optical flow to investigate the reproducibility of the motor response caused by local mechanical stimulation of the leech skin. We analyzed variability at different levels of processing: mechanosensory neurons, motoneurons, muscle activation, and behavior. Spike trains in mechanosensory neurons were very reproducible, unlike those in motoneurons. The motor response, however, was reproducible
\end{abstract}

Understanding the neural code by identifying the relevant features of the neural activity that are reproducible from trial to trial during the execution of a repeated behavior (Bialek and Rieke, 1992; Mainen and Sejnowski, 1995; Gerstner et al., 1997; Shadlen and Newsome, 1998; Stevens and Zador, 1998; Lestienne, 2001) requires both quantitative observation of the behavior and the simultaneous measurement of a large fraction of its underlying neural activity. Nervous systems of invertebrates, such as the leech (Muller et al., 1981), are particularly suitable models for such an investigation.

The CNS of the leech Hirudo medicinalis is composed of a chain of 21 segmental ganglia, each consisting of $\sim 400$ neurons (Nicholls and Baylor, 1968; Muller et al., 1981). Two pairs of roots, formed by the axons of mechanosensory neurons and motoneurons, emerge from each ganglion and innervate the corresponding body segment. Mechanosensory neurons have all been identified and electrophysiologically characterized (Muller et al., 1981). Most motoneurons have also been identified, and their role in mediating leech motor responses has been extensively studied (Stuart, 1970; Kristan, 1982; Lockery and Kristan, 1990a).

When the skin of the leech is touched, the animal bends its body to withdraw from the stimulus (Kristan, 1982; Lockery and Kristan, 1990a). This simple response, referred to as local bending, is initiated by a moderate mechanical stimulation and is primarily mediated by mechanosensory pressure (P) cells (Kristan, 1982; Lewis and Kristan, 1998a,b; Zoccolan and Torre, 2002), whose

\footnotetext{
Received July 3, 2002; revised Sept. 13, 2002; accepted Sept. 18, 2002.

This work was funded by European Union Grant Parallel 960211. We thank Dr. Hugh Robinson for valuable scientific suggestions and helpful comments on this manuscript and Manuela Schipizza Lough and Dylan Dean for editing the text.

Correspondence should be addressed to Vincent Torre, Scuola Internazionale Superiore di Studi Avanzati, Via Beirut 2, 34014 Trieste, Italy. E-mail: torre@sissa.it.

G. Pinato's present address: Department of Medical Physiology, University of Copenhagen, DK-2200 Copenhagen, Denmark.

Copyright (C) 2002 Society for Neuroscience $\quad 0270-6474 / 02 / 2210790-11 \$ 15.00 / 0$
}

because of two distinct biophysical mechanisms. First, leech muscles contract slowly and therefore are poorly sensitive to the jitter of motoneuron spikes. Second, the motor response results from the coactivation of a population of motoneurons firing in a statistically independent way, which reduces the variability of the population firing. These data show that reproducible spike trains are not required to sustain reproducible behaviors and illustrate how the nervous system can cope with unreliable components to produce reliable action.

Key words: sensorimotor responses; optical flow; statistical independence; pooling variability; population coding; reproducibility of muscle contraction

firing is highly reproducible (Pinato and Torre, 2000). The purpose of this work was to investigate (1) the reproducibility of the local bending and (2) the reproducibility of the neural coding of the local bending in the output stage (motoneurons) of the leech CNS.

The local bending was quantitatively characterized by computing the optical flow, whose usefulness in neurobiology we have demonstrated recently (Zoccolan et al., 2001). The firing activity of a large fraction of motoneurons involved in the behavior was measured by combining parallel extracellular recordings from the roots and spike sorting techniques (Ort et al., 1974; Pinato and Torre, 2000; Pinato et al., 2000; Arisi et al., 2001).

Our results show that the firing pattern of individual motoneurons is highly variable from trial to trial and that local bending is mediated by the coactivation of a population of motoneurons firing in a statistically independent way. Because of statistical independence, the electrical activity of the motoneuron population becomes less variable. In addition, the time course of muscle contraction primarily depends on the number of incoming motoneuron spikes and not on their exact timing. These mechanisms are the basis of motor output reproducibility in leech local bending.

We show here that reproducible motor output does not require reproducible firing of individual motoneurons and illustrate the biophysical mechanisms that allow a reproducible motor output to be driven by highly variable spike trains. Similar properties are likely to be general features of neural computation and widely present across nervous systems.

\section{MATERIALS AND METHODS}

Preparation and electrical recordings. Two preparations were used: the first to quantify skin deformation and the second to analyze motoneuron firing during local bending. Both preparations were kept in a Sylgardcoated dish at room temperature $\left(20-24^{\circ} \mathrm{C}\right)$ and bathed in Ringer's solution (in mM: $115 \mathrm{NaCl}, 1.8 \mathrm{CaCl}_{2}, 4 \mathrm{KCl}, 12$ glucose, and 10 Tris maleate buffered to $\mathrm{pH} 7.4$ with $\mathrm{NaOH}$ ) (Muller et al., 1981).

The first preparation consisted of a hemisection of leech skin approximately three segments in length, isolated from the rest of the body (Zoccolan et al., 2001). The skin was flattened and fixed with pins to the 
bottom of the recording chamber but was allowed to deform during muscle contraction. The middle segment was kept innervated by its ganglion.

The second preparation was an isolated leech segmental ganglion with exposed nerve roots. Five suction pipettes were used to perform parallel extracellular recordings from the anterior-anterior (AA), anteriormedial (MA), and posterior-posterior (PP) roots and from the two bifurcations of the dorsal posterior root (DP:B1 and DP:B2) (Stent et al., 1978; Pinato et al., 2000; Arisi et al., 2001). Spikes recorded from these roots were classified according to dimension and shape and were identified by impaling each motoneuron with a sharp intracellular microelectrode (input resistance, $30 \mathrm{M} \Omega$; filled with $4 \mathrm{M}$ potassium acetate), as described previously (Pinato et al., 2000; Arisi et al., 2001). In this way, it was possible to characterize the firing activity of a large fraction of all leech motoneurons: the excitatory motoneurons of longitudinal muscles (cells 3, 4, 5, 6, 8, 107, 108, and L), the excitor of flattener muscles (cell 109), the annulus erector, and two inhibitory motoneurons of longitudinal muscles (cells 102 and 119) (Ort et al., 1974; Stent et al., 1978; Arisi et al., 2001). All but one of the voltage signals of excitatory motoneurons of circular muscles were not identifiable in the extracellular recordings from the nerve roots. It was possible to monitor the activity of only one circular excitor: cell CiV.

In both preparations, local bending was initiated by intracellular stimulation of mechanosensory dorsal or ventral $\mathrm{P}$ cells, ipsilateral to the recorded roots. In some experiments, a mechanical stimulus was delivered to the first preparation by rapidly pressing a nylon filament driven by a solenoid on the skin, as described previously (Pinato and Torre, 2000).

Stimulation of individual motoneurons. Simultaneous extracellular recordings from the roots were also used to verify that electrical coupling between pairs of motoneurons (Ort et al., 1974) is not strong enough to induce a sustained firing in other motoneurons while stimulating a specific one. Seventy-seven intracellular stimulations of individual motoneurons were performed in five different preparations $(\sim 15$ motoneurons impaled in each experiment). Low-frequency $(10-15 \mathrm{~Hz})$ bursts in stimulated motoneurons were always unable to affect the spontaneous firing pattern of other simultaneously recorded motoneurons. Higherfrequency $(20-40 \mathrm{~Hz}$ ) and long-lasting (up to $1 \mathrm{sec}$ ) bursts in stimulated motoneurons generally evoked only a few spikes in other recorded motoneurons, in some cases transiently increasing their spontaneous firing rate 2 or $4 \mathrm{~Hz}$, although in 2 of the 77 cases, a high-frequency burst $(>40 \mathrm{~Hz})$ in the stimulated motoneuron increased the firing rate of a motoneuron coupled to it up to $\sim 15 \mathrm{~Hz}$. These data indicate that, in experiments in which skin deformations were evoked by stimulating an individual motoneuron (see Figs. 3, 5, 9), the contribution to the response by other motoneurons is minimal.

Imaging and behavior analysis. Skin deformations were quantified by computing the optical flows (see Fig. $1 B$ ) from image sequences of the contracting leech skin. Images were acquired at 5 or $8.3 \mathrm{~Hz}$ by a standard CCD camera mounted on a dissecting microscope and were then digitized and stored on a personal computer. The method for computing the optical flow is based on finding the best correlation between patches of successive images and is fully described in a previous work (Zoccolan et al., 2001). The same technique was used to follow the displacement of a specific point of the leech skin, as in Figure $1 D$. The optical flow is decomposed to its elementary components (see Fig. 1C) in two steps. First, the optical flow in a given window is approximated by a linear vector field; second, from this linear approximation, the elementary deformations are obtained (Zoccolan et al., 2001). The coefficient of variation $(\mathrm{CV})$ of the significant elementary deformations (see Fig. 1C) provides a compact and precise measure of the reproducibility of the pattern of skin deformation.

Spike train analysis. The variability of spikes fired by individual motoneurons was characterized by computing the $\mathrm{CV}$ of their firing rate over the number of trials of a repeated stimulation, in a given time window $\Delta t$. $\Delta t$ was chosen equal to $200 \mathrm{msec}$, because in this time window leech muscles integrate motoneuron spikes, and a noticeable skin contraction can be observed (Arisi et al., 2001). The spike variability of the population of coactivated motoneurons was quantified by computing the $\mathrm{CV}$ of the population firing rate, i.e., the sum of individual motoneuron firing rates. When motoneurons in the population fire in a statistically independent way, the $\mathrm{CV}$ of the population firing is significantly lower than the CV of individual motoneurons (Pinato et al., 2000; Arisi et al., 2001).

Statistical independence in motoneuron firing was measured by computing, for each pair of coactivated motoneurons $i$ and $j$, the following quantities: (1) the probability, $p_{i}$, that motoneuron $i$ fires at least one spike in a given time interval $\Delta t ;(2)$ the joint probability, $p_{i j}$ that both motoneurons $i$ and $j$ fire at least one spike in the same $\Delta t$; (3) the entropy for the activity of motoneuron $i$, defined as $H_{i}=-\Sigma_{m} P_{i}^{m} \log _{2} P_{i}^{m}$, where $P_{i}^{m}$ is the probability that motoneuron $i$ fires $m$ spikes in $\Delta t$; and (4) the joint entropy, $H_{i j}=-\Sigma_{m n} P_{i j}^{m n} \log _{2} P_{i j}^{m n}$, where $P_{i j}^{m n}$ gives the probability that motoneuron $i$ fires $m$ spikes and motoneuron $j$ fires $n$ spikes in $\Delta t$. When the joint probability of firing $P_{i j}$ is equal to the product $P_{i} / P_{j}$, and the joint entropy $H_{i j}$ is equal to the sum $H_{i}+H_{j}$, the firing of the two neurons is statistically independent (Pinato et al., 2000). Notice that entropy offers a more complete way to evaluate statistical independence than does joint probability, in that entropy takes into account all possible firing states (neuron $i$ firing $0,1,2, \ldots, m$ spikes) of the neuron, whereas $P_{1}$ only gives the probability that the neuron fired. Nevertheless, $P_{i}$ can be used to evaluate statistical independence if the probability for the neuron to fire more than one spike in $\Delta t$ is very low. For this reason, probabilities were computed for a time window of $20 \mathrm{msec}$, chosen for a low probability of firing more than one spike.

\section{RESULTS}

\section{Reproducibility of the local bending}

The firing pattern of mechanosensory neurons and the reproducibility of the local bending were studied using a leech skin preparation, in which a leech body wall segment was flattened over a piece of Sylgard while ganglion innervation was maintained (Nicholls and Baylor, 1968; Stuart, 1970; Kristan, 1982; Zoccolan et al., 2001; Zoccolan and Torre, 2002).

Figure $1 A$ shows the typical firing pattern of a dorsal $\mathrm{P}$ cell $\left(\mathrm{P}_{\mathrm{d}}\right)$ after mechanical stimulation of the dorsal body wall. When the skin was touched with a nylon filament, the cell $\mathrm{P}_{\mathrm{d}}$ quickly responded with highly reproducible firing. In fact, both the number of spikes and their timing were highly reproducible, with a jitter ranging from $<100 \mu \mathrm{sec}$ to $<4 \mathrm{msec}$ for the first four spikes fired by the neuron (Pinato and Torre, 2000).

The firing of mechanosensory neurons made muscle fibers contract and initiate local bending. The intensity and shape of local bending are almost completely determined by the $\mathrm{P}$ cells (Kristan, 1982; Zoccolan and Torre, 2002). Therefore, the motor response evoked by a controlled number of spikes in one of the $\mathrm{P}$ cells was studied. The associated maximal skin deformation was quantitatively characterized by computing the optical flow, which is a two-dimensional vector field that describes the point displacements on the skin and provides a complete characterization of the motor response (Zoccolan et al., 2001). The optical flow is obtained from image sequences of the contracting leech skin taken by a CCD camera, and the method to compute it has been fully described in a previous work (Zoccolan et al., 2001).

Figure $1 B$ shows the optical flow induced on the leech skin by evoking two spikes in the cell $\mathrm{P}_{\mathrm{d}}$. The flow is drawn on a gray background, showing the annular margins of the imaged skin preparation. The local structure of the optical flow can be analyzed by computing its best linear approximation in a region (Fig. $1 B$, box) around its stationary point (Fig. $1 B, \times)$. The linear approximation is completely characterized by six parameters: the coordinates of the stationary point and the four elementary deformations: rotation $(\omega)$, expansion $(E)$, horizontal shear $\left(S_{1}\right)$, and oblique shear $\left(S_{2}\right)$ (Zoccolan et al., 2001). The flow shown in Figure $1 B$ is a dorsal compression, sustained by coactivation of longitudinal and circular muscles (Zoccolan and Torre, 2002) and characterized by a high negative expansion $(E)$ and a significant positive shear $\left(S_{1}\right.$; Fig. $\left.1 C\right)$. The reproducibility of this motor response was quantified by computing the $\mathrm{CV}$ of its significant elementary deformations ( $E$ and $S_{1}$ ), which was $\sim 0.3$ (Fig. $1 C$, bottom). The variability of the motor response was also quantified by measuring the displacement of selected points on the leech 


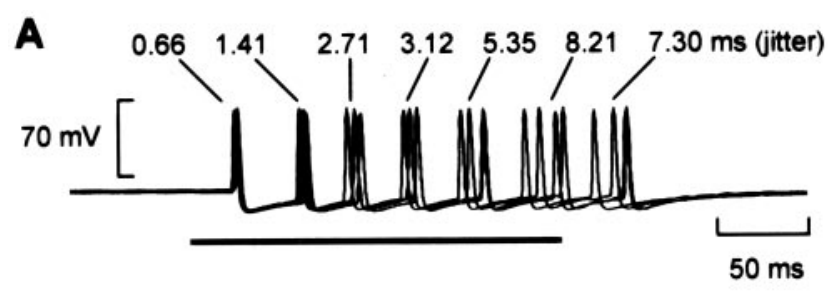

B
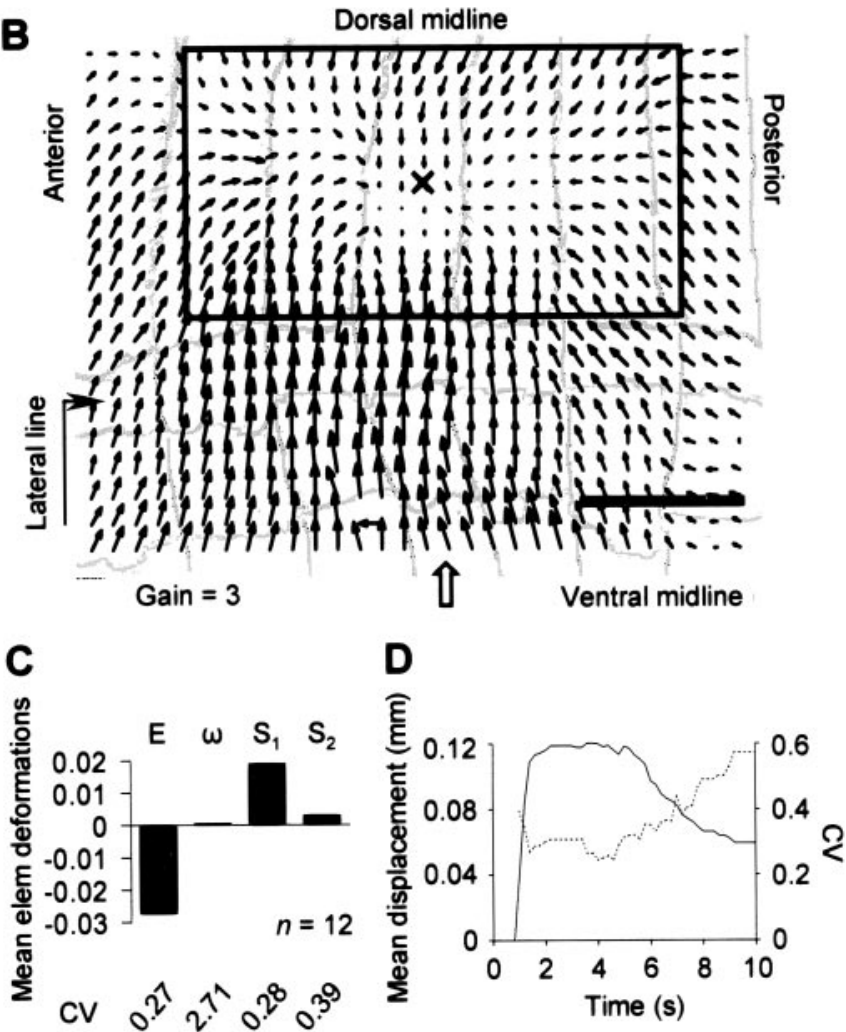

Figure 1. Reproducibility of local bending. A, Four superimposed intracellular recordings from a dorsal $\mathrm{P}$ cell after identical mechanical stimulation $(20 \mathrm{mN})$ of the dorsal leech skin. The thick bar indicates the stimulus duration $(200 \mathrm{msec})$. The number above each action potential is the jitter (i.e., the SD of latency from the stimulus onset) of that action potential in milliseconds. $B$, Optical flow describing the maximal skin deformation induced by evoking two action potentials in a dorsal $\mathrm{P}$ cell. The optical flow is computed on a grid of $30 \times 20$ points. The gray background shows the annular margins of the leech skin preparation. The central annulus of the innervated segment is indicated by the white arrow. The $\times$ indicates the stationary point, i.e., the point that stays at rest during the contraction. The region framed by the box was used to compute the linear approximation of the optical flow, from which the elementary deformations shown in $C$ were computed. To make the direction of the movement more clear, the field is drawn with a magnification of $3 \times . C$, Decomposition of the optical flow in the box of $B$ into the four elementary deformations: $E, \omega, S_{1}$, and $S_{2}$. The mean values of the elementary deformations over 12 trials of the same stimulation are drawn. Their CV is shown at the bottom. $D$, Time evolution of the displacement (solid line) and the corresponding $\mathrm{CV}$ (dotted line) of a representative point on the leech skin during the same trials of $C$.

skin and by measuring their CV (Fig. 1D). These points were usually selected at the edge of the region chosen to compute the linearization (Fig. 1B, box). On average, the $\mathrm{CV}$ of the point displacements was between 0.2 and 0.3 . These results were confirmed by experiments repeated on seven different preparations, in which the dorsal and the ventral $\mathrm{P}$ cells were alternatively stimulated and the skin was touched. All these experiments
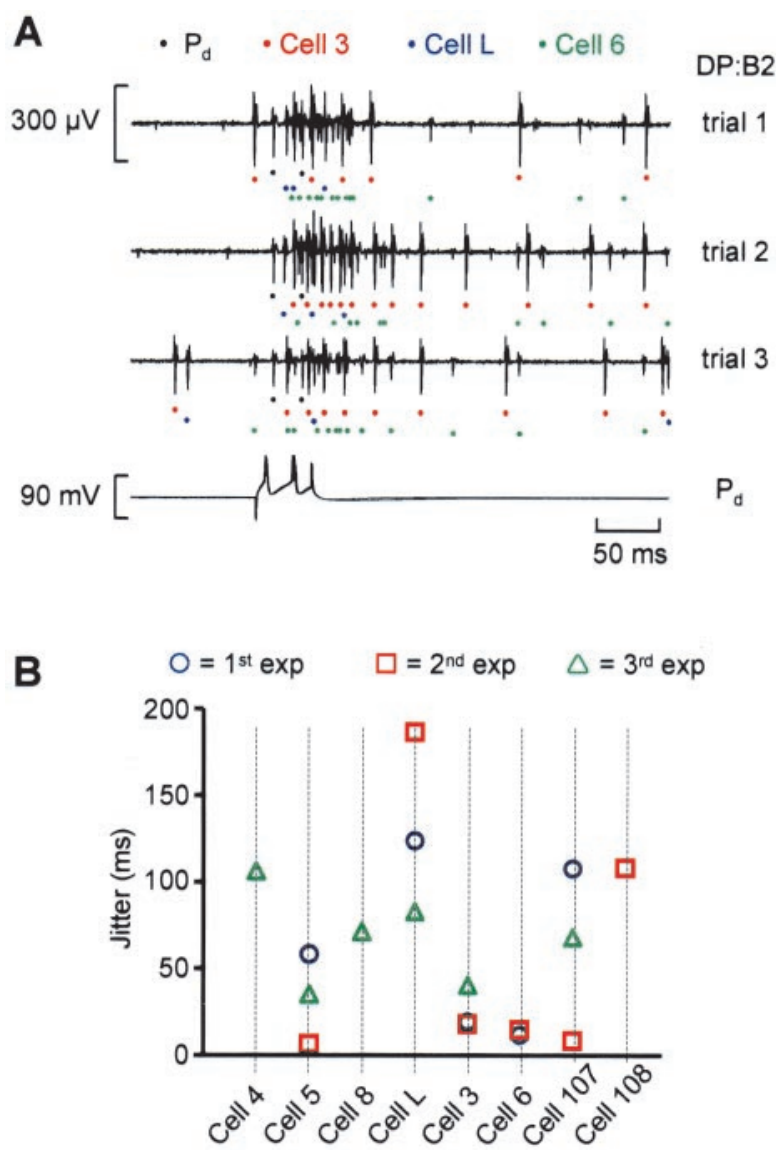

Figure 2. Variability of motoneuron spike trains during local bending. $A$, Three extracellular voltage recordings from the DP:B2 root during identical intracellular stimulation of a $\mathrm{P}$ cell with two spikes (bottom trace). In the extracellular recordings, the spikes from a $\mathrm{P}$ cell and from motoneurons $3, \mathrm{~L}$, and 6 are indicated by black, red, blue, and green dots, respectively. B, Blue circles, Jitter of the first spike of the motoneurons shown in $A$ and five other motoneurons simultaneously recorded from the AA, MA, PP, and DP:B1 roots. Red squares, green triangles, Similar data obtained from identical experiments repeated in two other preparations. In all three experiments considered, spike discharges in motoneurons were evoked by intracellular stimulation of a dorsal $\mathrm{P}$ cell with two spikes. Jitter was computed from 76,85 , and 44 repetitions of the same stimulation for experiments 1 (blue circles), 2 (red squares), and 3 ( green triangles), respectively. Motoneurons 4, 5, 8, L, 3, 6, 107, and 108 are all excitors of longitudinal muscles.

showed that the pattern of skin deformation during local bending is characterized by a $\mathrm{CV}$ between 0.2 and 0.3 in both its global shape and the displacement of single points on the skin surface.

\section{Timing of motoneuron spike trains and low-pass filter properties of muscle fibers}

Having assessed the reproducibility of the local bending (Fig. $1 C, D)$ and of its coding at the level of mechanosensory neurons (Fig. 1A) (Pinato and Torre, 2000), the next step of our analysis was to investigate the reproducibility of spike trains in motoneurons, i.e., in the ganglion output.

The firing pattern of motoneurons after P cell stimulation was studied in the isolated leech ganglion. By using extracellular suction pipettes, it was possible to record voltage signals from fine roots ipsilateral to the stimulated $\mathrm{P}$ cell and to precisely characterize the variability of motoneuron spike trains involved in local bending (see Materials and Methods).

Figure $2 A$ shows three extracellular recordings from the DP:B2 
bifurcation, obtained during identical repeated stimulation of the dorsal $\mathrm{P}$ cell (intracellular recording from $\mathrm{P}$ cell shown in Fig. $2 A$, bottom). Spikes fired by three identified motoneurons are indicated by rows of dots of different colors (green, motoneuron 6; blue, motoneuron L; red, motoneuron 3 ). These are all excitatory motoneurons innervating longitudinal muscle fiber, responsible for the shortening of the leech body during local bending. These motoneurons fired in a rather irregular way among different trials, and this result is emphasized by the comparison with the precise timing of the spikes fired by $\mathrm{P}$ cell (black dots). Figure $2 B$ shows the jitter of the first spike evoked in these motoneurons and in other five longitudinal excitatory motoneurons (blue circles), simultaneously recorded from the other roots. Data from similar experiments performed on two other preparations are also shown (red squares, green triangles). In every experiment, the firing pattern of all coactivated motoneurons was much more variable than the firing activity of mechanosensory neurons (compare Fig. 1A). The first spikes occurred with a jitter usually higher than $10 \mathrm{msec}$ (second experiment: motoneuron 5, $\sim 6 \mathrm{msec}$; motoneuron 107, $\sim 8$ msec; first and second experiments: motoneuron 3, $\sim 19$ msec; motoneuron $6, \sim 12 \mathrm{msec}$ ) and often of the order of 50-100 msec. For some motoneurons, the jitter was $>200 \mathrm{msec}$ (first experiment: motoneurons 4 and 8 , data not shown). The second spikes had a still higher jitter, whereas the third, fourth, and fifth spikes had jitters ranging from several hundred milliseconds to $>1000$ msec (data not shown).

These experiments were repeated in five different preparations (data from three shown in Fig. 2B), in which the dorsal and the ventral $\mathrm{P}$ cells were alternatively stimulated. As shown in Figure $2 B$, for some motoneurons the value of the jitter of the first and consecutive spikes had a large variability from preparation to preparation. In summary, all these experiments clearly showed that the precise time coding of the stimulus in mechanosensory neurons is not preserved at the level of the motoneurons. Nevertheless, the motor response elicited by local mechanical stimulation of the leech skin is significantly reproducible (Fig. 1C,D). One of the biophysical mechanisms that can account for the reproducibility of the local bending is the low-pass-filtering property of the muscles. Leech muscles (Mason and Kristan, 1982; Arisi et al., 2001; Zoccolan and Torre, 2002), as some muscles of other invertebrates and lower vertebrates (Morris and Hooper, 1997; Morris and Hooper, 1998), contract very slowly and integrate the incoming spike trains over a very long time window (several hundred milliseconds). This suggests that the time course of the muscle contraction primarily depends on the number of spikes fired by the activated motoneurons (Mason and Kristan, 1982; Morris and Hooper, 1997, 1998) and not on their exact timing of firing rate.

This integration property of the leech muscles was verified in a series of experiments in which the spike timing of a single motoneuron was changed by applying depolarizing current steps of different durations and intensities but that all evoked the same total number of action potentials. Figure $3 A$ shows the time course of the displacement of a selected point on the leech skin, when spike trains of different durations and frequencies were evoked in motoneuron 3 , an excitor of longitudinal muscles. The displacements indicated in Figure $3 A$ (dotted traces, solid traces) were induced by the spike discharges shown respectively in the top (first stimulation protocol) and the bottom (second stimulation protocol) of Figure $3 B$. The total number of spikes evoked by these two intracellular stimuli was the same (37), but their time distributions were completely different. Nevertheless, the result-

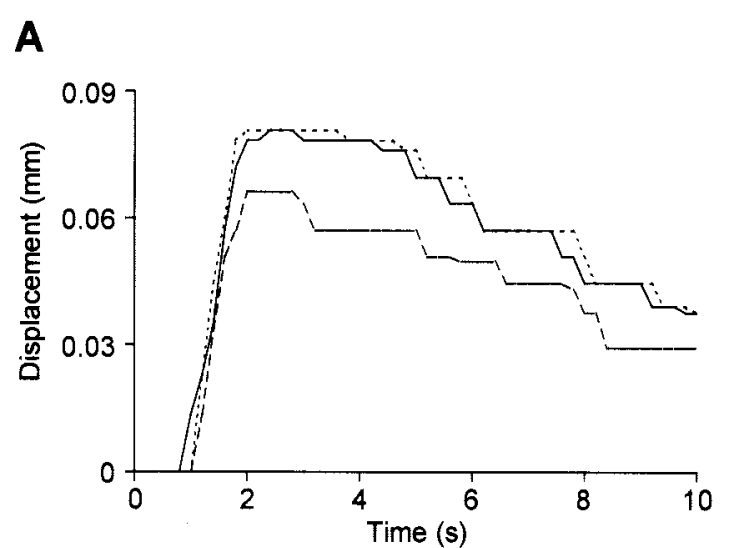

B
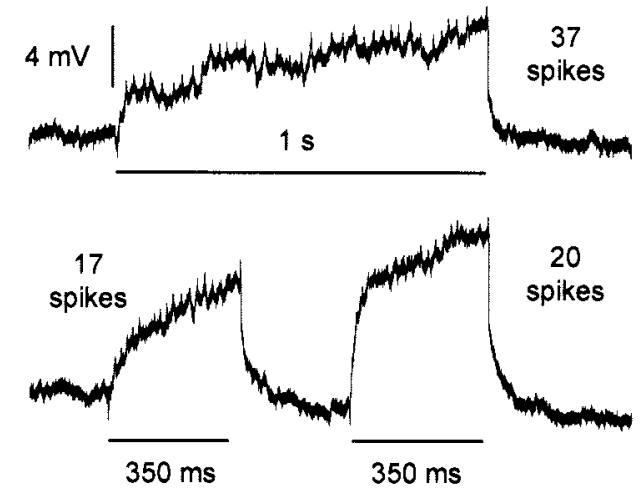

Figure 3. Low-pass filter properties of muscle fibers. $A$, Time course of the displacement of a selected point on the leech skin, when motoneuron 3 was forced to fire a controlled number of spikes (37) in three different stimulations. The displacements indicated by the dotted and solid traces were induced by the spike discharges shown respectively at the top and bottom of $B$. The displacement indicated by the dashed trace was evoked by a spike discharge similar to that shown in the top of $B . B, T o p$, Spike train evoked in motoneuron 3 by a single depolarizing current step of 0.7 nA lasting $1 \mathrm{sec}$ (total number of evoked spikes, 37). Bottom, Spike train evoked in motoneuron 3 by two consecutive depolarizing current steps of $1 \mathrm{nA}$ lasting $350 \mathrm{msec}$ each (total number of evoked spikes, 37).

ing displacements were almost identical. Note that muscle contraction has an intrinsic high variability, so that, when a spike train identical to that shown in Figure $3 B$, top, was again evoked, the corresponding displacement (Fig. $3 A$, dashed line) differed from the dotted trace more than the solid trace did (this property will be discussed in the next section). Data presented in Figure 3 show that contractions induced by the second protocols do not differ from contractions induced by the first more than what would be expected from the natural variability of muscle contraction evoked by a fixed number of spikes (see Fig. 5). Similar stimulation protocols, applied to five different motoneurons in three different preparations, confirmed the results shown in Figure 3. In all experiments, skin displacements evoked by the second protocol were always statistically indistinguishable by those evoked by the first one: (1) the second protocol produced displacements that were always within $1 \mathrm{SD}$ from the mean displacement induced by the first stimulation protocol; and (2) including data from the second protocol in the computation of mean and SD for the first did not significantly change the value of the mean or increase the SD. 


\section{Reproducibility of motoneuron firing}

Having assessed that muscle contraction primarily depends on the number of spikes fired by activated motoneurons, the reproducibility of motoneuron firing after mechanical stimulation was investigated. As shown in Figure 3, the contraction of leech muscles is not sensitive to the jitter of the motoneuron spikes, but muscle contraction may be sensitive to the number of the incoming spikes in the time window over which spikes are integrated. In fact, Figure $2 A$ shows that not only is the spike timing of coactivated motoneurons significantly variable, but the spike number is, too.

The statistical properties of motoneuron firing were quantified by computing the average firing rate (AFR) and its $\mathrm{CV}$ for all identified motoneurons coactivated during local bending. A 200 msec time bin was used, because this is large enough to observe a noticeable skin contraction when single motoneurons are induced to fire at a physiological rate (Arisi et al., 2001). Moreover, in the experiments performed to study local bending, muscle contraction developed $200 \mathrm{msec}$ after mechanosensory stimulation and reached its peak in $\sim 1 \sec ($ Fig. $1 D$ ). Therefore, muscle fibers integrate motoneuron spikes over an effective time window of $\sim 200-400 \mathrm{msec}$.

Figure 4 shows the AFR (solid line) and the CV (dashed line) for all identified motoneurons from the experiment already shown in Figure 2, $A$ and $B$ (blue circles). In the experiment, two spikes were evoked in the cell $\mathrm{P}_{\mathrm{d}}$. All motoneuron bursts were recorded from roots ipsilateral to the stimulated cell $\mathrm{P}_{\mathrm{d}}$. This means that the innervation fields of the recorded motoneurons were all ipsilateral to the receptive field of the cell $\mathrm{P}_{\mathrm{d}}$ (contralateral motoneurons were not recorded in the present study). As expected (Lockery and Kristan, 1990a; Zoccolan and Torre, 2002), all the identified dorsal excitors (DEs), i.e., the excitatory motoneurons of dorsal longitudinal muscles, were activated by the stimulus. The firing rate of these motoneurons (cells 6, 5, 107, and 3) increased to $15-30 \mathrm{~Hz}$ after $30-60 \mathrm{msec}$ (mean latency of the first spike) from the stimulus onset, with a CV transiently decreasing from $\sim 1$ to a value between 0.3 and 0.5 at the peak of the response. Other identified motoneurons were coactivated by the stimulus: cell L, excitor of all longitudinal muscles; cells 4 and 8 , ventral excitors (VEs); cell $\mathrm{CiV}$, an excitor of ventral circular muscles; cell 109, an excitor of flattener muscles (FEs); and cell 119, a ventral inhibitor (VI). These neurons were activated at lower rate $(5-10 \mathrm{~Hz})$ with a $\mathrm{CV}$ at the peak of the response just $<1$ for most of them. Other motoneurons were inhibited: cell 108 , a VE; cell 102, a dorsal inhibitor (DI).

This pattern of activation of motoneurons is consistent with the classical description of dorsal local bending: activation of all DEs and cell L, activation of VI, and inhibition of DI and VE (Lockery and Kristan, 1990a). It also confirms the involvement of motoneurons inducing transverse contractions ( $\mathrm{CiV}$ and $\mathrm{FE}$ ) in the local bending (Zoccolan and Torre, 2002). Figure 4 shows also that some VEs, such as cells 4 and 8, were excited. These neurons were activated at a low rate by the $P_{d}$ firing $(5-10 \mathrm{~Hz})$, whereas ventral $\mathrm{P}$ cell $\left(\mathrm{P}_{\mathrm{v}}\right)$ firing activated them at a much higher rate $(20-30 \mathrm{~Hz}$; data not shown). As a general trend, in all experiments performed (five), we observed that (1) DEs and VEs were always strongly activated by, respectively, $\mathrm{P}_{\mathrm{d}}$ and $\mathrm{P}_{\mathrm{v}}$ firing, and (2) some VEs and some DEs could be activated at a low rate by, respectively, $\mathrm{P}_{\mathrm{d}}$ and $\mathrm{P}_{\mathrm{v}}$ firing. These results suggest that some VEs and some DEs can be recruited during the stimulation of, respectively, dorsal and ventral $\mathrm{P}$ cells in a rather variable way
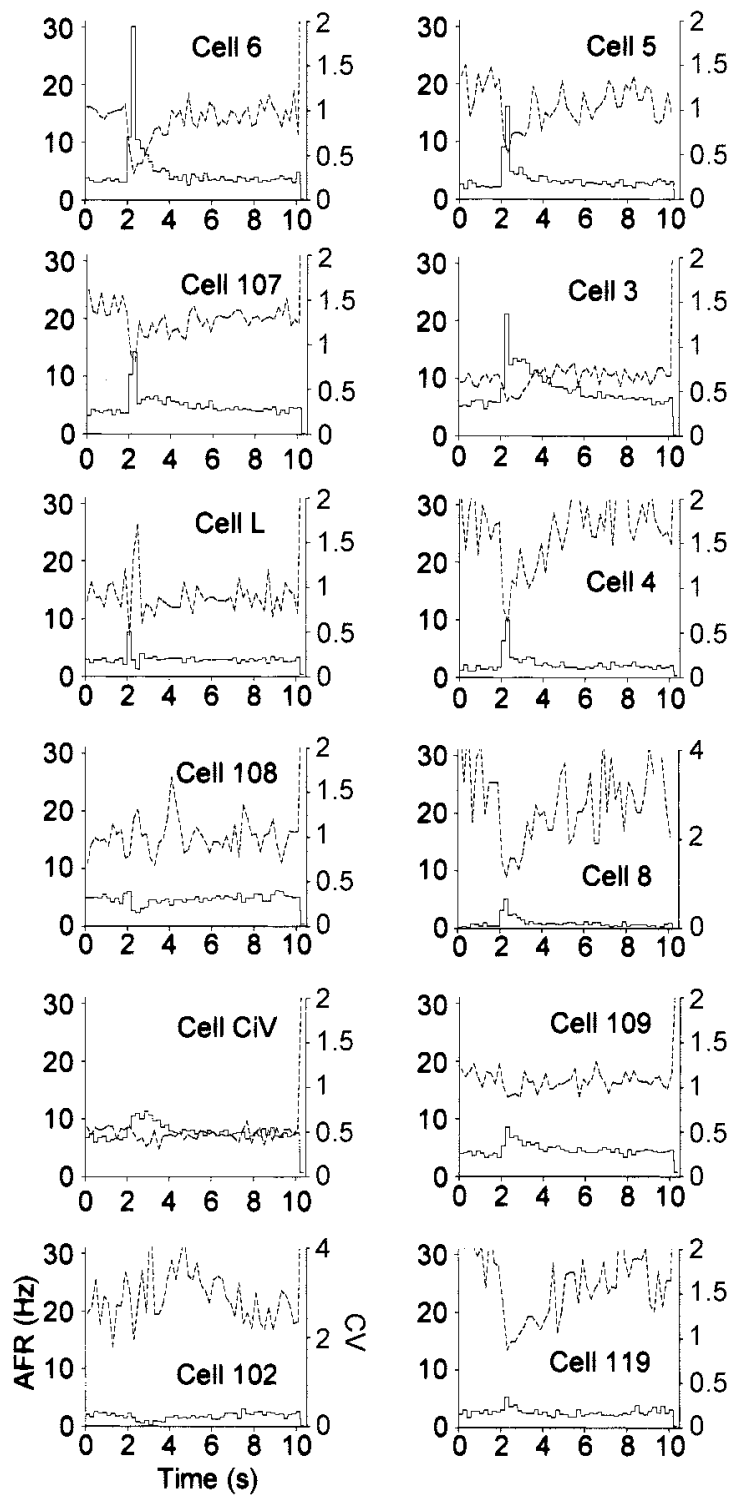

Figure 4. First-order statistics of motoneuron spike trains. Average firing rate (solid lines) and coefficient of variation (dashed lines) of the activity of 12 coactivated motoneurons during local bending are shown. Motoneurons are from the same experiment analyzed in Figure 2, $A$ and $B$ (blue circles). Local bending was elicited by inducing a dorsal $\mathrm{P}$ cell to fire two spikes. Data were obtained from 76 different trials. Bin width, $200 \mathrm{msec}$.

from preparation to preparation. This is consistent with the execution of lateral bending, which is usually elicited by simultaneous activation of both dorsal and ventral $\mathrm{P}$ cells on the same side of the ganglion (Lockery and Kristan, 1990a,b; Kristan et al., 1995).

Figure 4 shows that the $\mathrm{CV}$ of the firing activity of individual motoneurons coactivated during local bending is rather high. All activated motoneurons have a CV between 0.4 and 1 at the peak of the response, with the only exception being cell $6(\mathrm{CV} \sim 0.3)$. Similar results were obtained in all five tested preparations, in which the dorsal and ventral P cells were alternatively stimulated. In every experiment, between 8 and 12 motoneurons were simultaneously recorded and identified, and their AFR and CV were computed. Almost all recorded motoneurons had a CV between 0.4 and 1 at the peak of the AFR. No more than one dorsal or 

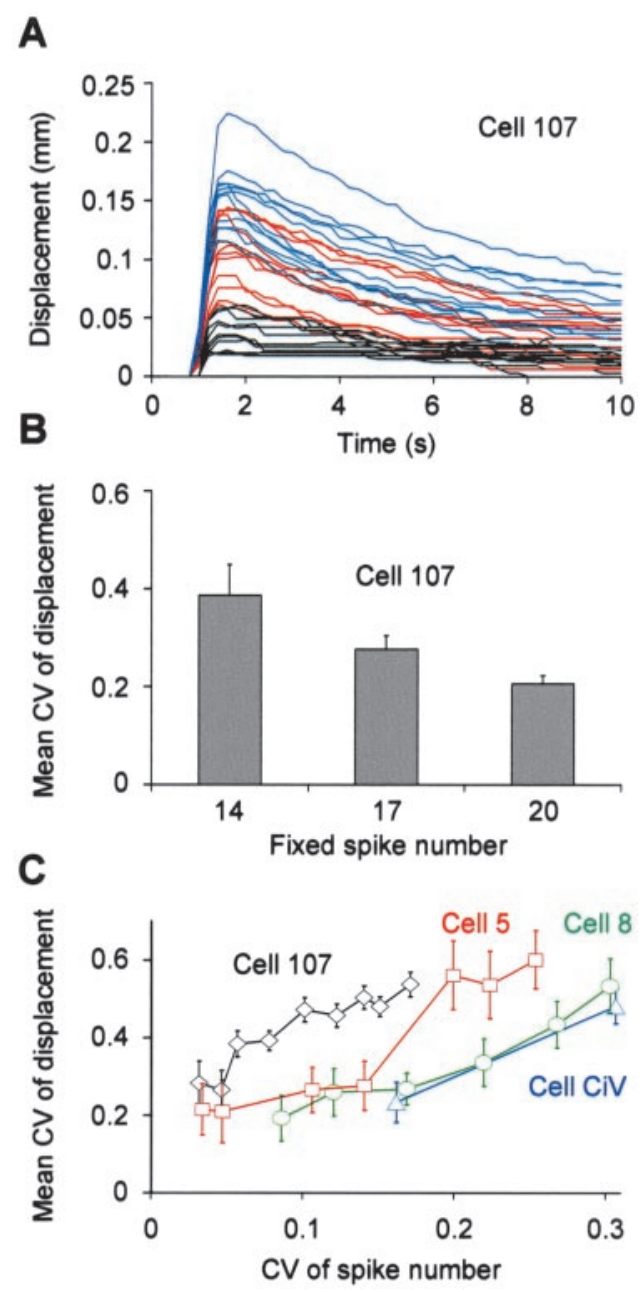

Figure 5. Variability of muscle contraction. $A$, Displacement of a selected point on the leech skin during the repetitive stimulation of motoneuron 107 with depolarizing current steps of increasing size, lasting $400 \mathrm{msec}$ and evoking a controlled number of spikes in the neuron. Eleven trials (black lines) with 14 spikes, 12 trials (red lines) with 17 spikes, and 11 trials (blue lines) with 20 spikes were recorded. B, Mean CV of the maximal displacement of 6 selected points, when a fixed number of spikes were evoked in motoneuron 107 in different repetitions. Eleven, 12, and 11 repetitions with 14,17 , and 20 spikes, respectively, were considered. $C$, Relationship between the mean CV of the maximal displacement of 6 selected points and the $\mathrm{CV}$ of the spike number for motoneurons 107 (black diamonds), 5 (red squares), 8 (green circles), and $\mathrm{CiV}$ (blue triangles) impaled in four different preparations.

ventral excitor per experiment had a $\mathrm{CV}$ of $\sim 0.3$ after stimulation, respectively, of the dorsal or ventral $\mathrm{P}$ cell. To test whether such variable firing rates in individual motoneurons can sustain the reproducible motor responses observed during local bending (Fig. 1C,D), we investigated the reproducibility of the contractions induced by single-motoneuron firing.

\section{Variability of muscle contraction}

The variability of the motor response depends on the variability of the motoneuron firing pattern as well as on the variability of the muscle contraction (see Discussion; Stuart, 1970; Hoover et al., 2002). The variability of muscle contraction was studied by impaling individual motoneurons with a sharp intracellular electrode and by evoking a controlled number of spikes. The displacement of a representative point on the skin was followed when 14 (Fig. 5A, black traces), 17 (red traces), or 20 (blue traces) spikes

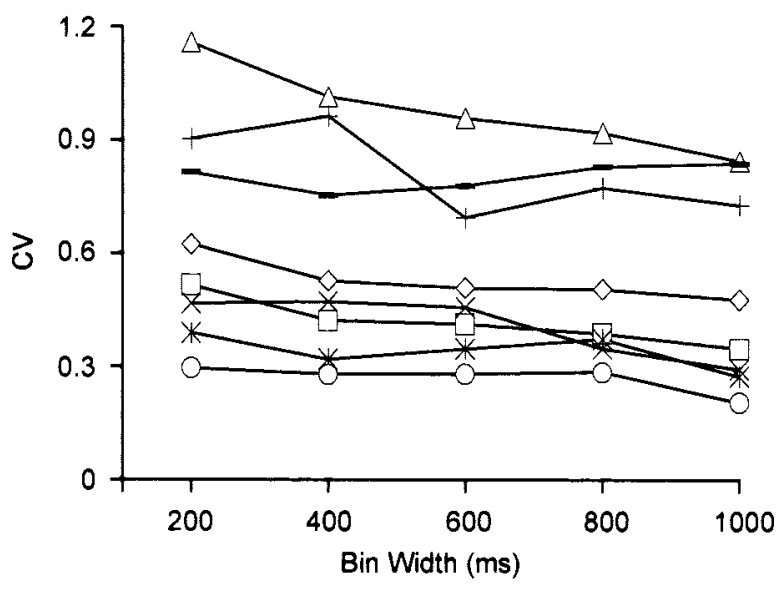

Figure 6. Variability of spike number in motoneuron bursts. Coefficients of variation of the spike number, in time bins of increasing size after $\mathrm{P}_{\mathrm{d}}$ firing, are shown for motoneurons 6 (circles), 3 (asterisks), L $(\times), 5$ (squares), 4 (diamonds), $108(+), 107(-)$ and 8 (triangles) (same data as in Fig. 4).

were evoked within $400 \mathrm{msec}$ in motoneuron 107 by depolarizing current steps of increasing size. The $\mathrm{CV}$ of the associated displacement for a fixed number of spikes was $\sim 0.4$ for 17 spikes and decreased to $\sim 0.2$ for 20 or more spikes (Fig. $5 B$ ). When the number of spikes of the motoneuron was allowed to vary, the $\mathrm{CV}$ of the skin displacement increased almost linearly with the $\mathrm{CV}$ of the motoneuron firing, as shown in Figure 5C (black line). Figure $5 C$ also shows a similar trend for three other motoneurons in three different preparations: cell 5 (red line), cell 8 (green line), and cell $\mathrm{CiV}$ (blue line). These results, repeated in four different preparations, show that the $\mathrm{CV}$ measured for the local bending, between 0.2 and 0.3 (Fig. $1 C, D$ ), is just larger than the $\mathrm{CV}$ of the skin deformation evoked by a single motoneuron firing with a $\mathrm{CV}$ of almost 0, i.e., with almost perfect reproducibility (Fig. 5B). From the data shown in Figure $5 C$, we expect that a $\mathrm{CV}$ of the skin displacement between 0.2 and 0.5 should be produced by a single motoneuron firing with a CV varying between 0.1 and 0.2 . Single motoneurons whose firing rate has a $\mathrm{CV}$ of $\geq 0.3$ cannot induce skin deformations with a $\mathrm{CV}$ of $<0.5$.

Figure 4 suggests that the $\mathrm{CV}$ of the firing rate of coactivated motoneurons, at the peak of the response, is too high to account for the reproducibility of local bending. However, for slowcontracting, nonspiking muscles, such as those of the leech, the contraction amplitude primarily depends on the number of spikes in the motoneuron burst induced by mechanosensory stimulation rather than on the firing frequency of motoneurons (Morris and Hooper, 1997). Figure 6 shows the CV of the spike number, in time bins of increasing size after $\mathrm{P}_{\mathrm{d}}$ firing, for the first eight motoneurons analyzed in Figure 4. The time bin varies from 200 msec (the same as Fig. 4) to $1 \mathrm{sec}$, because local bending reached its peak in $\sim 1 \mathrm{sec}$ by the onset of the P cell stimulation (Fig. 1D), and the more active motoneurons (Fig. 4, first four panels) fired at sustained rate for $\sim 1-1.2 \mathrm{sec}$. Counting spikes in time windows of increasing size slightly reduced the variability in the number of spikes fired by activated motoneurons (Fig. 6). Nevertheless, even when very long time windows $(1 \mathrm{sec})$ were taken into account, the $\mathrm{CV}$ of the spike number of just one motoneuron (cell 6) reached a value as low as 0.2. All other motoneuron bursts had a CV of $\geq 0.3$ : cells 3 and $\mathrm{L}, \sim 0.3$; cell $5, \sim 0.35$; cell $4, \sim 0.5$; and cells 108 , 107 , and $8,>0.7$. 
Figure 7. Statistical independence of motoneuron firing. Joint probabilities and joint entropies are shown for all possible pair-wise combinations of motoneurons $6,5,3,107$, and 4 , whose AFR and CV are shown in Figure 4. Top right panels, For each pair of motoneurons $i$ and $j$, the joint probability of firing $p_{i j}$ (red line) is compared with the product of individual probabilities $p_{i} / p_{j}$ (black dashed line). Bottom left panels, The joint entropy $H_{i j}$ (red line) is compared with the sum of individual entropies $H_{i}+H_{j}$ (black dashed line). Bin width, 20 msec.

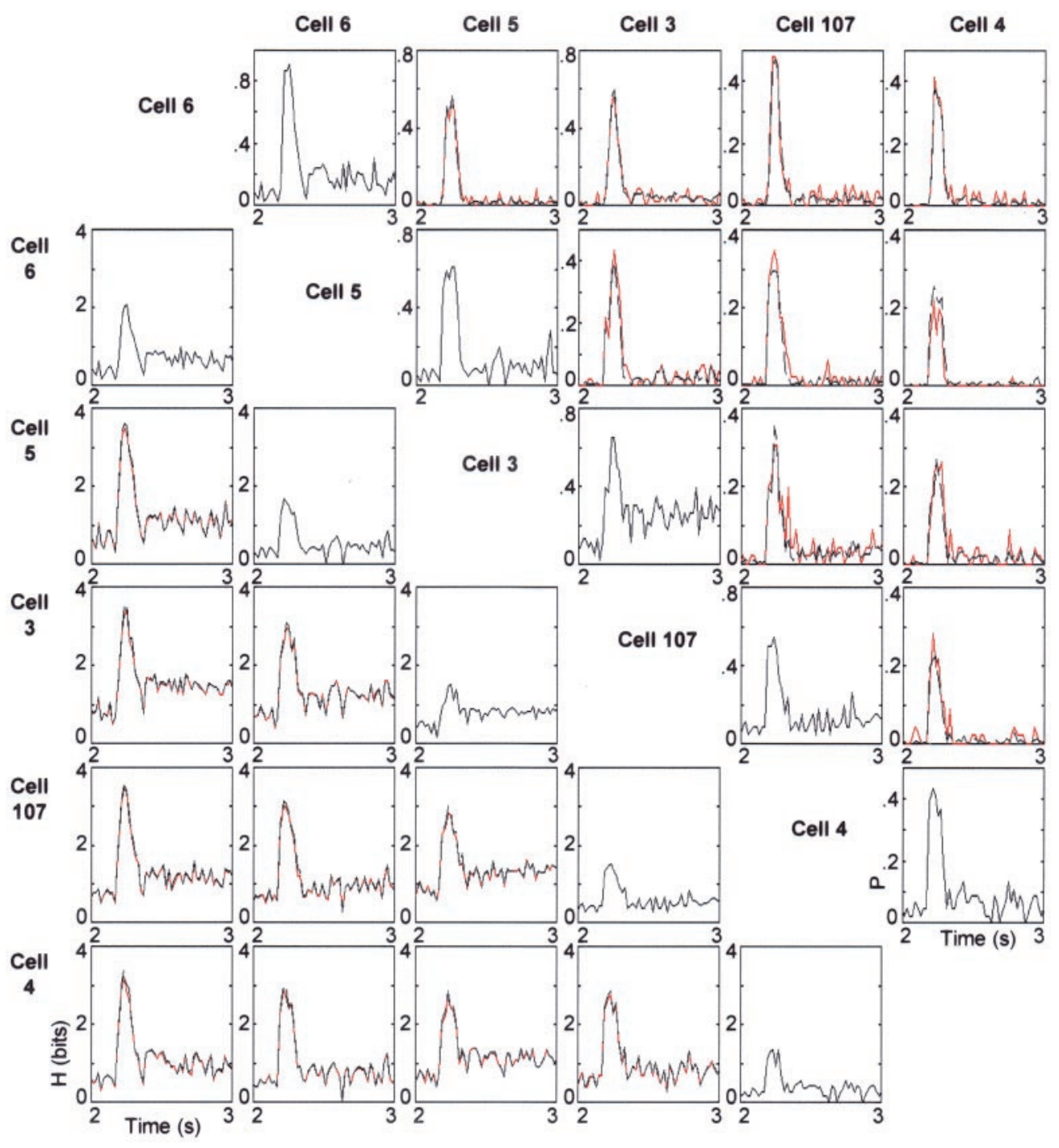

Data presented in Figures 4-6 clearly show that the reproducibility of the local bending cannot be exclusively explained by the low-pass-filtering properties of the leech muscles (Fig. 3). Although muscle fibers are not sensitive to the timing of the incoming spikes, muscle contractions are rather sensitive to variations in the spike number (Fig. 5C). The AFR of individual motoneurons, coactivated during local bending (Fig. 4), is definitely too variable to account for this reproducible behavior. Moreover, the number of spikes in motoneuron bursts lasting up to $1 \mathrm{sec}$, is also too variable to explain the reproducibility of the local bending (Fig. 6 ). Some other mechanism should take place to reduce variability of the motor output.

\section{Distributed organization of the motor output}

A biophysical mechanism that can explain such a low variability of the local bending is the distributed organization of the firing activity sustaining it at the level of the motoneurons. Figure 7 shows the analysis of statistical independence for the activity of some motoneurons whose AFR and CV were reported in Figure 4. The statistical independence of motoneuron firing was characterized by computing, for each pair of coactivated motoneurons, the joint probability, $p_{i j}$ that both motoneurons fire one spike, and $H_{i j}$, the joint entropy of their firing activity (Pinato et al., 2000). For all pairs of motoneurons shown in Figure 7 (the most strongly activated during local bending) (Fig. 4); $p_{i j}$ was almost equal to $p_{i} / p_{j}$ and $H_{i j}$ was identical to $H_{i}+H_{j}$ (see Materials and Methods). Thus, the firing of these motoneurons, coactivated during local bending, was pair-wise statistically independent. We found similar results for every pair of motoneurons shown in Figure 4 and for every pair of motoneurons recorded in all five experiments we performed.

These observations suggest that the motor response analyzed in Figure 1 has a distributed organization (Tsau et al., 1994; Wu et al., 1994; Lewis and Kristan, 1998a; Pinato et al., 2000; Arisi et al., 2001); it is produced by the coactivation of a population of motoneurons, and its low variability is the result of the underlying neuron pooling. This is supported by Figure $8 A$, in which the CVs of the firing rates of all identified longitudinal motoneurons activated during local bending (first eight neurons of Fig. 4) are drawn (dashed lined) together with the $\mathrm{CV}$ of their ensemble activity (solid line). Figure $8 A$ clearly shows that the $\mathrm{CV}$ of each individual motoneuron in the pool is significantly higher than that of the pooled activity, which is rarely $>0.5$ and has a minimum close to 0.2 at the peak of the response. A similar result was obtained when the number of spikes in motoneuron bursts was counted and its CV was computed (Fig. 6). The number of spikes of the population of coactivated motoneurons had a CV always 


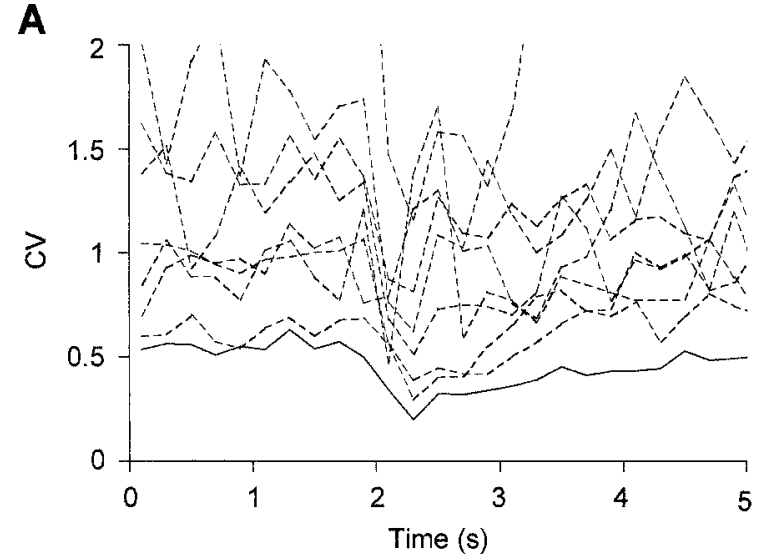

B

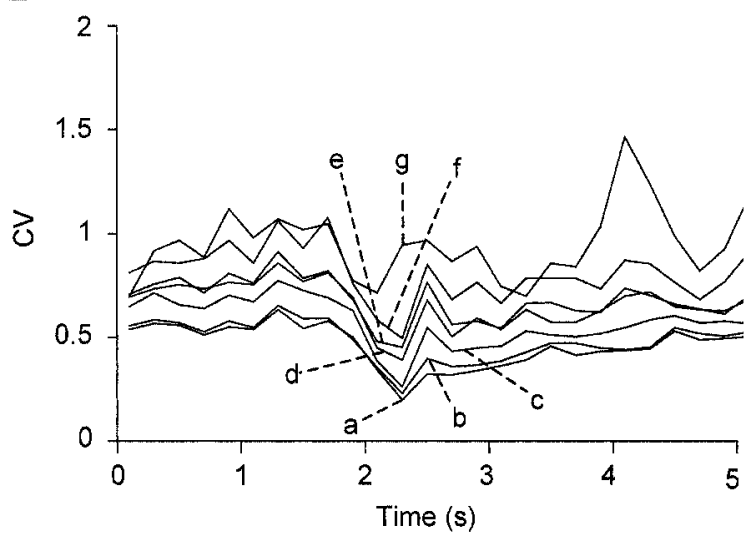

Figure 8. Pooling variability of coactivated motoneurons. $A$, The $\mathrm{CV}$ of eight longitudinal motoneurons (cells 6, 5, 107, 3, L, 4, 108, and 8) coactivated during local bending (dashed lines) is compared with the CV (solid line) of their pooled activity. Motoneurons are from the same experiment analyzed in Figures 2, 4, and 6. B, The CV of the pooled activity of the same motoneurons is computed by removing individual motoneurons one at the time from the pool. The bottom trace $(a)$ is the CV computed for the whole population of motoneurons (cells 6, 5, 107, 3, L, 4, 108, and 8) and corresponds to the solid line in $A$. The other traces are the CVs obtained by removing the following motoneurons from the starting population: cell 6 $(b)$; cells 6 and $3(c)$; cells 6,3 , and $5(d)$; cells $6,3,5$, and $4(e)$; cells 6,3 , 5,4 , and $\mathrm{L}(f)$; and cells $6,3,5,4, \mathrm{~L}$, and $107(\mathrm{~g})$.

close to 0.2 for every selected time window (from 200 msec up to $1 \mathrm{sec}$ after $\mathrm{P}_{\mathrm{d}}$ firing, in steps of $200 \mathrm{msec}$ ). This increase in reproducibility of the population firing is a consequence of the statistical independence illustrated in Figure 7 (Pinato et al., 2000). The pattern of activation of motoneurons after dorsal and ventral $\mathrm{P}$ cell firing was analyzed in five different preparations. All these experiments gave results similar to those shown in Figures 4,7 , and $8 A$; motoneurons fired quite irregularly from trial to trial, but the $\mathrm{CV}$ of their pooled activity had a minimum $<0.2$ because of their statistical independence. On the basis of data shown in Figure 5, this $\mathrm{CV}$ is small enough to guarantee a motor response as reproducible as that shown in Figure $1 D$.

This distributed coding across a population of independent motoneurons not only assures reliability of the motor response but also allows graceful degradation of performance with damage to individual motoneurons. We tested the robustness of population coding by computing the $\mathrm{CV}$ of the pooled activity of the motoneuron population from which data of single motoneurons were removed one at the time. The result is shown in Figure $8 B$. The CV increased in little steps while individual motoneurons were removed from the pool. This guarantees a gradual decrease in the reliability of motor output in case of damage to individual motoneurons. Note in particular that even when we removed the motoneurons with more reproducible firing (cells 6 and 3 ) from pooling, the variability of the population firing did not significantly increase and still had a CV with a minimum of $<0.25$ (for details, see legend to Fig. 8).

\section{Local bending is sustained by the coactivation of several motoneurons}

Given that local bending is sustained by the coactivation of a population of motoneurons (Fig. 4), and that skin deformations induced by pairs of motoneurons superimpose linearly (Zoccolan and Torre, 2002), we tested whether local bending can be approximated by the linear sum of an ensemble of skin deformations induced by individual motoneurons. We addressed this issue in a series of experiments in which we impaled and stimulated the $\mathrm{P}$ cells and as many motoneurons as possible in the same skin preparation.

Figure 9 summarizes the results of one of these experiments. Figure $9 A$ shows the optical flow induced by firing two spikes in a dorsal P cell. In the same preparation, motoneurons L, 3, 5, 112, and 107 were impaled. The corresponding optical flows are drawn in Figure $9 B-E$ (optical flow of cell 107 not shown). These optical flows were obtained by inducing the impaled motoneurons to fire a burst of action potentials at $15-20 \mathrm{~Hz}$ for $400 \mathrm{msec}$ to evoke firing patterns similar to those recorded during $\mathrm{P}_{\mathrm{d}}$ stimulation (Fig. 4). Figure $9 F$ shows the linear superposition of these optical flows induced by individual motoneurons. Because most excitors of longitudinal muscles were included in the sum, a marked similarity between the intensity and shape of the local bending (Fig. 9A) and those of the linear sum of the impaled motoneurons (Fig. $9 F$ ) is apparent. This was confirmed by calculating the elementary deformations of the two fields drawn in Figure 9, $A$ and $F$. As shown in Figure 10, the main elementary deformations $\left(E\right.$ and $S_{1}$ ) of these two optical flows differ for $<5 \%$. These results confirm that the local bending cannot be accounted for by the activation of just one motoneuron; it is necessary to consider the optical flows associated with a large number of motoneurons to obtain a good approximation of its shape and intensity. Specifically, Figure 9 shows that the coactivation of several motoneurons assures not only the reproducibility of the local bending (Fig. 8) but also its strength (note that the optical flows induced by motoneurons are shown with a magnification four times higher than the optical flow associated with the local bending). These results were confirmed in two other experiments in which a P cell and three or four motoneurons were impaled in the same preparation.

\section{DISCUSSION}

The data presented here show that local bending (a simple sensorimotor response of the leech) is rather reproducible (Fig. 1), but the firing of the motoneurons that sustains it is much more variable (Figs. 2, 4). Because it was possible to record simultaneously from the majority of motoneurons involved in the motor response (Fig. 4), the existence of hidden motoneurons firing in a highly reproducible way is unlikely. We therefore conclude that highly reproducible spike trains are not necessary for highly reproducible motor responses.

\section{Temporal averaging}

Reliability of the behavior is obtained by temporal and ensemble averaging. Temporal averaging is guaranteed by the low-pass- 
A

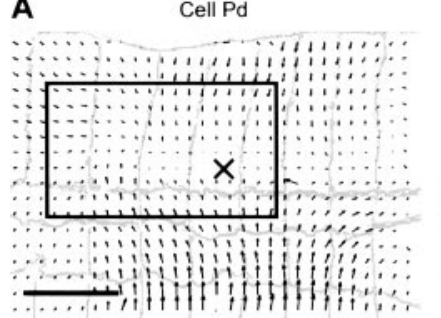

Figure 9. Distributed motor output. $A$ Optical flow describing the maximal skin contraction evoked by the firing of two spikes by a dorsal $\mathrm{P}$ cell. $B-E$, Optical flows describing the maximal skin contractions induced by the firing of motoneurons $\mathrm{L}, 3,5$, and 112, respectively, at $\sim 10-20 \mathrm{~Hz}$ in the same preparation. $F$, Linear superposition of the optical flows in $B-E$. The gain of the optical flow in $A$ and $F$ is 1 ; otherwise it is 4. The boxes in $A$ and $F$ are the linearization regions used to compute the stationary point position $(\times)$ and the elementary deformations shown in Figure 10.

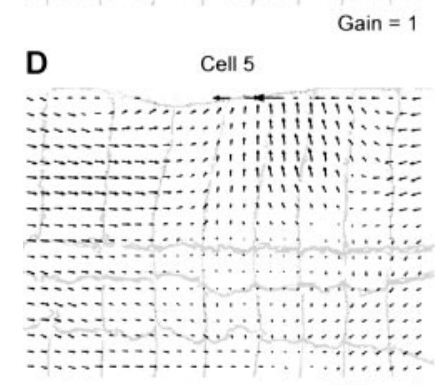

Gain $=4$

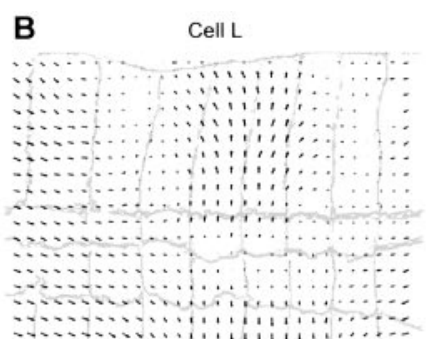

Gain $=4$

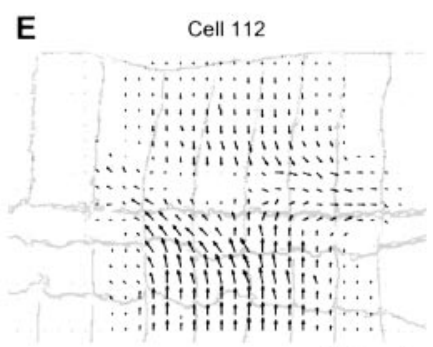

Gain $=4$

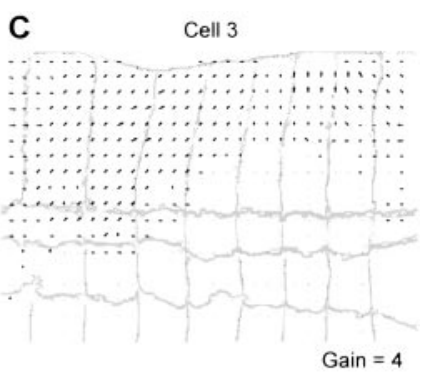

F Cells $\mathrm{L}+3+5+107+112$

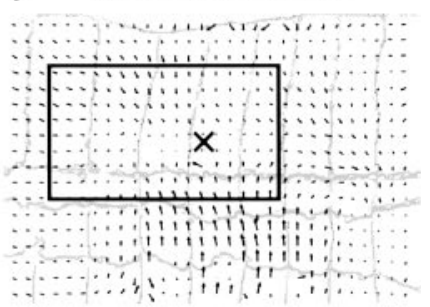

Gain $=1$

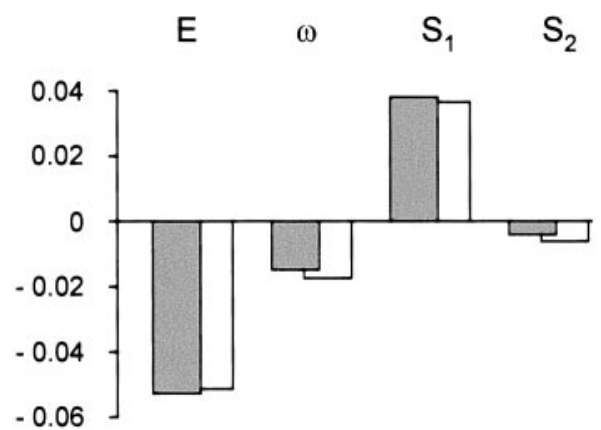

Figure 10. Accuracy of the linear superposition. Elementary deformations are shown for the optical flows shown in Figures 9, A (gray bars) and F (white bars).

filtering properties of the leech muscle fibers, which integrate motoneuron spikes within an effective time window of $\sim 200 \mathrm{msec}$ (Arisi et al., 2001) and make the time course of a contraction relatively insensitive to the jitter of motoneuron spikes.

Muscle contractions produced by individual motoneurons show significant variability even when driven by perfectly reliable spike trains (Fig. 5B). When spike trains in a motoneuron have a $\mathrm{CV}$ of $\sim 0.25$, the resulting muscle contraction has a $\mathrm{CV}$ of approximately or $>0.5$, i.e., significantly higher than the $\mathrm{CV}$ of the movements observed during local bending (compare Figs. 1, 5). Therefore, the reliability of local bending cannot be accounted only by the low-pass properties of muscle contraction.

\section{Ensemble averaging}

The motor response is mediated by coactivation of an ensemble of distinct motoneurons (Figs. 4, 7, 8) that fire in an almost statistically independent way (Fig. 7). Statistical independence compensates for the fluctuations (Figs. 4, 6) in the firing of individual motoneurons; thus the ensemble firing of the motoneuron population becomes less variable (Fig. $8 A$ ). In addition, fluctuations of muscle contraction evoked by individual motoneurons (Fig. 5) will probably be compensated and averaged by statistical independence (Hoover et al., 2002).

Figure 11 shows a simple scheme, indicating where, in the reflex pathway, variability is introduced and which biophysical mechanisms counteract it. The first spikes in the mechanosensory $\mathrm{P}$ cell

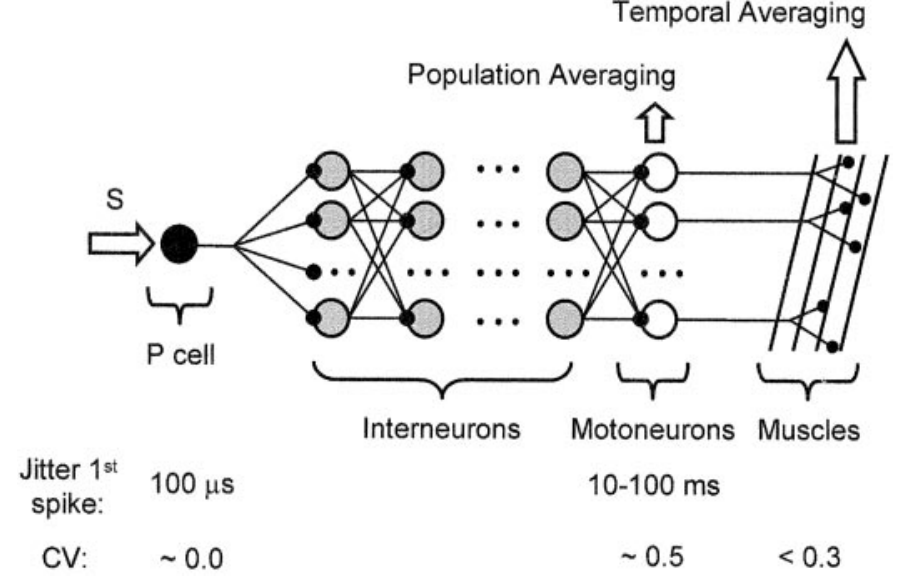

Figure 11. Proposed scheme of the neuronal network mediating local bending reflex. Mechanosensory stimuli $(S)$ are coded by precise firing of a pressure $\mathrm{P}$ cell (black unit). Then, neural signals cross some layers of interneurons ( gray units) to reach motoneurons (white units) that, in turn, innervate muscle fibers. The typical jitter of the first spike for $\mathrm{P}$ cells and motoneurons is shown at the bottom together with the CV of $\mathrm{P}$ cell firing, the typical $\mathrm{CV}$ of individual motoneuron firing, and the $\mathrm{CV}$ of the amplitude of the motor response. Reliability of the motor response is achieved by integrating spikes over a population of some dozens of motoneurons and over a time window of several hundred milliseconds.

are remarkably reproducible (Fig. $1 A$ ), often with a jitter of $<100$ $\mu \mathrm{sec}$ (Pinato and Torre, 2000). In a leech ganglion, neural signals from sensory neurons reach motoneurons through monosynaptic and polysynaptic pathways (Muller et al., 1981; Lockery and Kristan, 1990b; Wittenberg and Kristan, 1992). Polysynaptic pathways introduce significant variability, and the first spikes in motoneurons occur with a jitter of $>10 \mathrm{msec}$ and often on the order of $100 \mathrm{msec}$ (1000 times larger than in mechanosensory neurons). Given these biophysical constraints, reliability of the motor response in the leech is achieved by integrating spikes over a time window of several hundreds of milliseconds and over a population of some dozens of motoneurons. Therefore, population coding in the leech is used not only for interpolation between neurons with different receptive fields (Lewis and Kristan, 1998a) or population vector codes (Georgopoulos et al., 1986; Lewis and 
Kristan, 1998a; Georgopoulos, 2000) but also for increasing the reliability of the behavior. Indeed, if local bending were not mediated by a motoneuron population but only by a single motoneuron, given the high variability of individual motoneuron firing (Fig. 4) and the variability of muscle contraction (Fig. 5), the resulting motor response would have a $\mathrm{CV}$ of $\sim 0.6-0.7$, approximately three times that observed (Fig. 1). Because we are recording from the majority of leech motoneurons, it is highly unlikely that there is an undetected mechanism with more precise spikes.

\section{Comparison with previous studies}

A number of studies focused on reliability of neural firing in invertebrate (Bialek and Rieke, 1992; de Ruyter van Steveninck et al., 1997; Warzecha and Egelhaaf, 1999; Warzecha et al., 2000; Hoover et al., 2002) and mammalian (Shadlen and Newsome, 1994, 1998; Mainen and Sejnowski, 1995; Stevens and Zador, 1998; Kara et al., 2000; Lestienne, 2001) nervous systems, as well as on population coding (Georgopoulos et al., 1986; Lewis and Kristan, 1998a; Georgopoulos, 2000), correlated and uncorrelated firing (Zohary et al., 1994; Alonso et al., 1996; Raz et al., 1996; Bergman et al., 1998; Bair et al., 2001; Goldberg et al., 2002), and distributed organization of the neural response (Tsau et al., 1994; Wu et al., 1994; Middlebrooks et al., 1998). The present work describes, for the first time to our knowledge, how the reliability of the spike trains degrades, crossing consecutive stages of a nervous system during the execution of a simple behavior, and which biophysical mechanisms the nervous system uses to obtain global reliability with unreliable components. The stimulus is coded by precisely timed spikes in sensory neurons of the input stage, but the associated motor response is mediated by a rate code distributed across a population of statistically independent output units. This distributed rate code not only increases reliability of the behavior but also stabilizes the dynamics of the motor response, assures its robustness, and allows graceful degradation of performance with damage to individual motoneurons (Fig. 8B).

\section{The cat visual system}

A similar attempt to track variability at different neural stages has been performed recently in the cat visual system by Kara et al. (2000). They recorded simultaneously from three different stages of the cat nervous system and showed that trial-by-trial response variability of visual cells becomes higher at each consecutive visual stage. The present work and that of Kara et al. (2000) substantially differ in the choice of the model nervous system studied (invertebrate vs mammal) and of the investigated neural process (coding of sensorimotor responses versus representation of visual stimuli). Nevertheless, the results reported in the two studies are in general agreement, because both works show that neural responses become more variable as they cross consecutive synaptic levels.

\section{The stomatogastric neuromuscular system of the lobster}

A recent article by Hoover et al. (2002) was more strictly related to the present work. They recorded simultaneously (1) the extrajunctional potentials (EJPs) evoked in the pyloric muscles of the lobster stomatogastric neuromuscular system by motor nerve stimulation and (2) the amplitude of the pyloric muscle contraction. They found high variability in EJP amplitude, whereas the contraction of the corresponding pyloric muscle was highly deterministic. The issue they investigated, i.e., how electrical responses with large amplitude variation give rise to deterministic muscle output, is clearly related to the present work. However, several points make the present work and that of Hoover et al. (2002) significantly different. They studied the contraction of an individual muscle induced by stimulation of an individual motor nerve, whereas we investigated neural coding of a sensorimotor response by a population of coactivated sensory and motor neurons. The electrical responses they measured were EJPs in an activated muscle, whereas we recorded the firing pattern of tens of coactivated motoneurons, each innervating specific muscle fibers. In brief, the results obtained by Hoover et al. (2002) can be properly compared only with the results showing variability of the contraction induced by single-leech motoneuron firing, as reported in Figure 5 of this work. This comparison suggests that variability in the amplitude of EJPs could play similar roles in leech (Stuart, 1970) and lobster pyloric muscles and could be responsible for the variability of the contraction produced by reliable firing of single leech motoneurons (Fig. 5). Another mechanism proposed by Hoover et al. (2002) to reduce pyloric contraction variability, combinatorial averaging of EJPs in individual muscle fibers, could play a similar role in the leech muscles. This is suggested by the reduction in variability we observed when the number of spikes in the burst fired by a single motoneuron was increased (Fig. 5B). Finally, averaging across muscle fibers whose electrical responses are uncorrelated, the second mechanism proposed by Hoover et al. (2002) to reduce pyloric contraction variability, supports the idea that statistical independence in the electrical response of coactivated motoneurons (present work), individual muscle fibers (Hoover et al., 2002), or both is a powerful means to enhance reliability and stability of muscle contraction.

\section{Conclusions}

Averaging action potentials over an ensemble of neurons and over an appropriate time window is a likely mechanism for nervous systems to achieve high reproducibility despite using poorly reliable components such as neurons, synapses, and muscles. The extent of ensemble and time averaging, however, will be constrained by the task to be performed and by the biophysical properties of the specific neurons involved. The precision of spike occurrence depends on the requirements of the action or output: in the present case, a significant jitter (even of $\sim 100 \mathrm{msec}$ ) and a $\mathrm{CV}$ of $\sim 0.4$ in individual motoneurons were tolerated. Several tasks performed by the auditory system (Heil, 1997; Heil and Irvine, 1997; Middlebrooks et al., 1998; Furukawa and Middlebrooks, 2002) require very precise timing among spikes with a jitter of $<100 \mu \mathrm{sec}$. These data suggest that neurons are reliable on the time scale needed to attain the observed behavioral criteria. Therefore, any analysis of reliability of spike trains in a neuron or a population of neurons has to take into account the precision requirements imposed by the action or output to be produced.

\section{REFERENCES}

Alonso JM, Usrey WM, Reid RC (1996) Precisely correlated firing in cells of the lateral geniculate nucleus. Nature 383:815-819.

Arisi I, Zoccolan D, Torre V (2001) Distributed motor pattern underlying whole-body shortening in the medicinal leech. J Neurophysiol 86:2475-2488.

Bair W, Zohary E, Newsome WT (2001) Correlated firing in macaque visual area MT: time scales and relationship to behavior. J Neurosci $21: 1676-1697$.

Bergman H, Feingold A, Nini A, Raz A, Slovin H, Abeles M, Vaadia E (1998) Physiological aspects of information processing in the basal ganglia of normal and parkinsonian primates. Trends Neurosci 21:32-38. 
Bialek W, Rieke F (1992) Reliability and information transmission in spiking neurons. Trends Neurosci 15:428-434.

de Ruyter van Steveninck RR, Lewen GD, Strong SP, Koberle R, Bialek W (1997) Reproducibility and variability in neural spike trains. Science 275:1805-1808.

Furukawa S, Middlebrooks JC (2002) Cortical representation of auditory space: information-bearing features of spike patterns. J Neurophysiol 87:1749-1762

Georgopoulos AP (2000) Neural aspects of cognitive motor control. Curr Opin Neurobiol 10:238-241.

Georgopoulos AP, Schwartz AB, Kettner RE (1986) Neuronal population coding of movement direction. Science 233:1416-1419.

Gerstner W, Kreiter AK, Markram H, Herz AV (1997) Neural codes: firing rates and beyond. Proc Natl Acad Sci USA 94:12740-12741.

Goldberg JA, Boraud T, Maraton S, Haber SN, Vaadia E, Bergman H (2002) Enhanced synchrony among primary motor cortex neurons in the 1-methyl-4-phenyl-1,2,3,6-tetrahydropyridine primate model of Parkinson's disease. J Neurosci 22:4639-4653.

Heil P (1997) Auditory cortical onset responses revisited. I. First-spike timing. J Neurophysiol 77:2616-2641.

Heil P, Irvine DR (1997) First-spike timing of auditory-nerve fibers and comparison with auditory cortex. J Neurophysiol 78:2438-2454.

Hoover NJ, Weaver AL, Harness PI, Hooper SL (2002) Combinatorial and cross-fiber averaging transform muscle electrical responses with a large stochastic component into deterministic contractions. J Neurosci 22:1895-1904.

Kara P, Reinagel P, Reid RC (2000) Low response variability in simultaneously recorded retinal, thalamic, and cortical neurons. Neuron 27:635-646.

Kristan Jr WB (1982) Sensory and motor neurones responsible for the local bending response in leeches. J Exp Biol 96:161-180.

Kristan Jr WB, Lockery SR, Lewis JE (1995) Using reflexive behaviors of the medicinal leech to study information processing. J Neurobiol 27:380-389.

Lestienne R (2001) Spike timing, synchronization and information processing on the sensory side of the central nervous system. Prog Neurobiol 65:545-591.

Lewis JE, Kristan Jr WB (1998a) A neuronal network for computing population vectors in the leech. Nature 391:76-79.

Lewis JE, Kristan Jr WB (1998b) Representation of touch location by a population of leech sensory neurons. J Neurophysiol 80:2584-2592.

Lockery SR, Kristan Jr WB (1990a) Distributed processing of sensory information in the leech. I. Input-output relations of the local bending reflex. J Neurosci 10:1811-1815.

Lockery SR, Kristan Jr WB (1990b) Distributed processing of sensory information in the leech. II. Identification of interneurons contributing to the local bending reflex. J Neurosci 10:1816-1829.

Mainen ZF, Sejnowski TJ (1995) Reliability of spike timing in neocortical neurons. Science 268:1503-1506.

Mason A, Kristan Jr WB (1982) Neuronal excitation, inhibition and modulation of leech longitudinal muscle. J Comp Physiol 146:527-536.

Middlebrooks JC, Xu L, Eddins AC, Green DM (1998) Codes for soundsource location in nontonotopic auditory cortex. J Neurophysiol 80:863-881.

Morris LG, Hooper SL (1997) Muscle response to changing neuronal input in the lobster (Panulirus interruptus) stomatogastric system: spike number- versus spike frequency-dependent domains. J Neurosci 17:5956-5971.

Morris LG, Hooper SL (1998) Muscle response to changing neuronal input in the lobster (Panulirus interruptus) stomatogastric system: slow muscle properties can transform rhythmic input into tonic output. J Neurosci 18:3433-3442.

Muller KJ, Nicholls JG, Stent GS (1981) Neurobiology of the leech. New York: Cold Spring Harbor Laboratory.

Nicholls JG, Baylor DA (1968) Specific modalities and receptive fields of sensory neurons in CNS of the leech. J Neurophysiol 31:740-756.

Ort CA, Kristan Jr WB, Stent GS (1974) Neuronal control of swimming in the medicinal leech II. Identification and connections of motor neurons. J Comp Physiol [A] 94:121-154.

Pinato G, Torre V (2000) Coding and adaptation during mechanical stimulation in the leech nervous system. J Physiol (Lond) 529:747-762.

Pinato G, Battiston S, Torre V (2000) Statistical independence and neural computation in the leech ganglion. Biol Cybern 83:119-130.

Raz A, Feingold A, Zelanskaya V, Vaadia E, Bergman H (1996) Neuronal synchronization of tonically active neurons in the striatum of normal and parkinsonian primates. J Neurophysiol 76:2083-2088.

Shadlen MN, Newsome WT (1994) Noise, neural codes and cortical organization. Curr Opin Neurobiol 4:569-579.

Shadlen MN, Newsome WT (1998) The variable discharge of cortical neurons: implications for connectivity, computation, and information coding. J Neurosci 18:3870-3896.

Stent GS, Kristan Jr WB, Friesen WO, Ort CA, Poon M, Calabrese RL (1978) Neuronal generation of the leech swimming movement. Science 200:1348-1357.

Stevens CF, Zador AM (1998) Input synchrony and the irregular firing of cortical neurons. Nat Neurosci 1:210-217.

Stuart AE (1970) Physiological and morphological properties of motoneurones in the central nervous system of the leech. J Physiol (Lond) 209:627-646.

Tsau Y, Wu JY, Hopp HP, Cohen LB, Schiminovich D, Falk CX (1994) Distributed aspects of the response to siphon touch in Aplysia: spread of stimulus information and cross-correlation analysis. J Neurosci 14:4167-4184.

Warzecha AK, Egelhaaf M (1999) Variability in spike trains during constant and dynamic stimulation. Science 283:1927-1930.

Warzecha AK, Kretzberg J, Egelhaaf M (2000) Reliability of a fly motion-sensitive neuron depends on stimulus parameters. J Neurosci 20:8886-8896.

Wittenberg G, Kristan Jr WB (1992) Analysis and modeling of the multisegmental coordination of shortening behavior in the medicinal leech. II. Role of identified interneurons. J Neurophysiol 68:1693-1707.

Wu JY, Cohen LB, Falk CX (1994) Neuronal activity during different behaviors in Aplysia: a distributed organization? Science 263:820-823.

Zoccolan D, Torre V (2002) Using optical flow to characterize sensorymotor interactions in a segment of the medicinal leech. J Neurosci 22:2283-2298.

Zoccolan D, Giachetti A, Torre V (2001) The use of optical flow to characterize muscle contraction. J Neurosci Methods 110:65-80.

Zohary E, Shadlen MN, Newsome WT (1994) Correlated neuronal discharge rate and its implications for psychophysical performance. Nature 370:140-143. 